\title{
Gleason Score and Laterality Concordance between Prostate Biopsy and Prostatectomy Specimens
}

\author{
Kenneth G. Nepple, Terry L. Wahls, Stephen L. Hillis, Fadi N. Joudi \\ Department of Urology (KGN, FNJ), University of Iowa, Iowa City, Department of Surgery (FNJ), \\ Veterans Affairs, Iowa City Health Care System, Center for Research in the Implementation of Innovative \\ Strategies in Practice (TLW, SLH), Iowa City, Iowa, USA
}

\begin{abstract}
Objectives: Prostate biopsy involvement and Gleason score guide treatment decisions in prostate cancer. We evaluated concordance in Gleason score and laterality between biopsy and radical retropubic prostatectomy (RRP) specimens and factors that influenced this relationship.

Materials and Methods: We reviewed 538 prostate cancer diagnoses at a Veterans Affairs medical center (2000-2005) to identify men with prostate biopsy and RRP specimens. During this time there was a move from limited (6 core) to extended (12 core) biopsy schemes. Discordance in Gleason score was defined as any change in Gleason score.

Results: 152 men underwent RRP with biopsy showing Gleason $<7$ in 56\%, 7 in 36\%, and $>7$ in $8 \%$. Biopsy involvement was unilateral in 59\% and bilateral in 41\%. Compared to the biopsy, RRP Gleason score was concordant in 76 (50\%), higher in 51 (34\%), and lower in 25 (16\%). Bilateral involvement was concordant in 97\%, while unilateral involvement was concordant in only $20 \%$. Both Gleason score and laterality were concordant in only $26 \%$. Gleason concordance was higher in those with 8 or more cores compared to $<8$ cores taken $(54 \%$ vs. $34 \%, p=0.046)$, but concordance was not affected by age, PSA, prostate volume, or length of time from biopsy to RRP. During later years, concordance did not improve despite taking more cores.

Conclusions: Prostate biopsy underestimated prostatectomy Gleason score in 34\% of men and bilateral involvement in $80 \%$ of those with unilateral disease on biopsy. Taking at least eight cores improves the accuracy of the prostate biopsy.
\end{abstract}

Key words: prostate neoplasms; biopsy; prostatectomy

Int Braz J Urol. 2009; 35: 559-64

\section{INTRODUCTION}

Prostate cancer is common in men with an estimated 218,890 new diagnoses and 27,050 deaths in 2007 (1). The pathologic diagnosis of prostate cancer is based on transrectal ultrasound guided prostate biopsy which provides information on Gleason grade and unilateral versus bilateral cancer involvement. Gleason grade and prostate biopsy involvement subsequently guide treatment decisions, and influence surgical decisions with respect to nerve-sparing and pelvic lymph node dissection at the time of radical retropubic prostatectomy (RRP). Because of a trend in the PSA era toward patients presenting with low PSA levels and nonpalpable disease, pretreatment Gleason score may be the most important prognostic factor for treatment response and patient outcome (2).

It is important for clinicians to have information regarding the accuracy of prostate biopsy specimens. Prior studies have suggested that taking more prostate cores may improve the concordance between biopsy and prostatectomy (2-5), while others have not 
found such a relationship (6). Our main study objective was to evaluate concordance of Gleason score and laterality between prostate biopsy and RRP specimens in a cohort of our patients. A secondary objective was to evaluate whether an increased number of cores improved concordance.

\section{MATERIALS AND METHODS}

We retrospectively reviewed 538 prostate cancer diagnoses at the Veterans Affairs Medical Center Iowa City from 2000-2005 to identify men who underwent RRP. Patients were excluded if prostatectomy was not performed or if pathology reports from both prostate biopsy and RRP specimens were not available. Prostate biopsies were obtained using transrectal ultrasound guidance and the number of cores taken was at the discretion of the clinician. Clinical and pathologic data was reviewed for information including pathology reports from prostate biopsy and RRP. PSA value prior to biopsy was used for analysis. Number of days from initial biopsy to RRP was calculated for each patient. We defined concordance as an exact match in grade (or laterality) from biopsy to corresponding RRP specimen. Discordance was defined as a difference in grade (or laterality) from biopsy to RRP (i.e. upgrading from Gleason $3+4$ to $4+3$ was considered discordant).

Analysis was performed using the chi-square or Fisher's exact test for categorical variables and the Wilcoxon rank sum test for ordinal or continuous variables. $\mathrm{P}<0.05$ was considered significant. Statistical analysis was performed using SigmaStat 3.5. Institutional Review Board approval for the study was obtained.

\section{RESULTS}

Of the 538 men diagnosed with prostate cancer during the index period (2000-2005), 152 met inclusion criteria and underwent RRP (Table-1). Prostate biopsy was Gleason $<7$ in $56 \%, 7$ in $36 \%$, and $>7$ in $8 \%$. Biopsy involvement was unilateral in $90(59 \%)$ and bilateral in $62(41 \%)$. During the time period of this study, there was a general move from limited (6 core) to extended (12 core) biopsy schemes (Figure-1).

Average age at RRP was 61.3 years (median 61.2 , range 44.1 to 74.5 ). Compared to biopsy, RRP Gleason score was concordant in $76(50 \%)$, upgraded in $51(34 \%)$, and downgraded in $25(16 \%)$. Of those 51 patients who had Gleason score upgraded on RRP, Gleason grade was increased by $<1$ grade in 7,1 grade in 39 , and $>1$ grade in 5 patients. In the 25 patients who had Gleason score downgraded on RRP, Gleason grade was decreased by $<1$ grade in 10,1 grade in 13 , and $>1$ grade in 2 patients.

Concordance was not affected by age, PSA, prostate volume, or length of time from biopsy to RRP (Table-2). However, differences were noted for Gleason sum 7 biopsies, as Gleason $3+4$ concordance was $67 \%$ (30 of 45 ) with upgrading in $22 \%$ ( 10 of 45 ) and downgrading in $11 \%$ (5 of 45) versus Gleason $4+3$, which was concordant in none (0 of 10$)$ with upgrading in $20 \%$ ( 2 of 10 ) and downgrading in $80 \%$ $(8$ of 10$)(p<0.001)$.

Gleason concordance was higher in those with 8 or more cores compared to $<8$ cores taken $(54 \%$ vs.

Table 1 - Patient characteristics.

\begin{tabular}{lc}
\hline & Overall \\
& $(\mathrm{N}=152)$ \\
\hline Age at diagnosis (mean) & 61.1 \\
Biopsy PSA (mean) & 6.72 \\
TRUS volume (mean) & 30.5 \\
Biopsy Gleason grade (mean) & 6.6 \\
Days biopsy to RRP (mean) & 81.1 \\
Biopsy Gleason score (N, \%) & \\
$3+2$ & $1(0.7 \%)$ \\
$3+3$ & $84(55.3 \%)$ \\
$3+4$ & $45(29.6 \%)$ \\
$4+3$ & $10(6.6 \%)$ \\
$4+4$ & $4(2.6 \%)$ \\
$4+5$ & $2(1.3 \%)$ \\
$5+4$ & $3(2.0 \%)$ \\
$5+5$ & $3(2.0 \%)$ \\
\hline
\end{tabular}

$T R U S=$ transrectal ultrasound $; R R P=$ radical retropubic prostatectomy. 


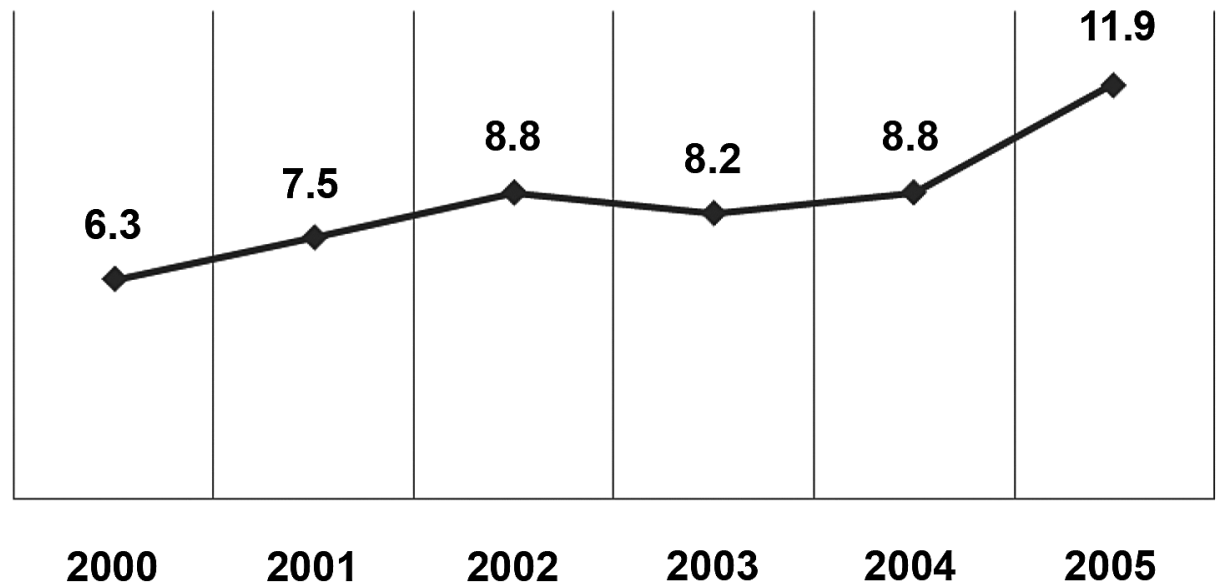

Figure 1 - Mean prostate core biopsies per patient.

$34 \%, \mathrm{p}=0.046)$. Table- 3 shows the concordance rate stratified by number of cores. The highest accuracy rate was in the group with 8-9 cores, with a statistically significant improvement in accuracy from 34\% to $64 \%$ seen when going from 5-7 to 8-9 cores $(\mathrm{p}=$ $0.03)$. Despite the increase in cores taken per patient in each subsequent year during our study (Figure-1), an incremental increase in accuracy over time was not observed (Table-4).

When biopsy involvement was unilateral, 72 of $90(80 \%)$ actually had bilateral involvement on RRP (Figure-2). In patients with bilateral biopsy involvement, 60 of $62(97 \%)$ had bilateral involvement on prostatectomy. Both Gleason score and laterality were concordant in only $26 \%$.

\section{COMMENTS}

Findings on prostate biopsy can differ from findings on RRP, and accurate assessment of prostate cancer on biopsy is important because it guides treatment decisions. Patients with low-grade prostate cancer are more likely to be offered expectant management without active treatment (7). Patients with higher grade involvement on prostate biopsy are more likely to have pelvic lymph node dissection performed and less likely to be offered nerve-sparing prostatectomy. We evaluated patients who underwent prostatectomy, and found that prostate biopsy underestimated Gleason score in 34\% of men and bilateral involvement in $80 \%$ of those with unilateral disease

Table 2 - Analysis of factors in patients with concordant vs. discordant Gleason grade from prostate biopsy to radical retropubic prostatectomy.

\begin{tabular}{lccc}
\hline & $\begin{array}{c}\text { Concordant } \\
(\mathrm{N}=76)\end{array}$ & $\begin{array}{c}\text { Discordant } \\
(\mathrm{N}=76)\end{array}$ & p Value \\
\hline Age at diagnosis (mean) & 60.6 & 61.5 & 0.21 \\
Biopsy PSA (mean) & 6.25 & 7.2 & 0.50 \\
TRUS volume (mean) & 29.9 & 31.1 & 0.32 \\
Biopsy Gleason grade (mean) & 6.4 & 6.7 & 0.18 \\
Days biopsy to RRP (mean) & 87.3 & 74.9 & 0.48 \\
\hline
\end{tabular}

$T R U S=$ transrectal ultrasound; $R R P=$ radical retropubic prostatectomy. 


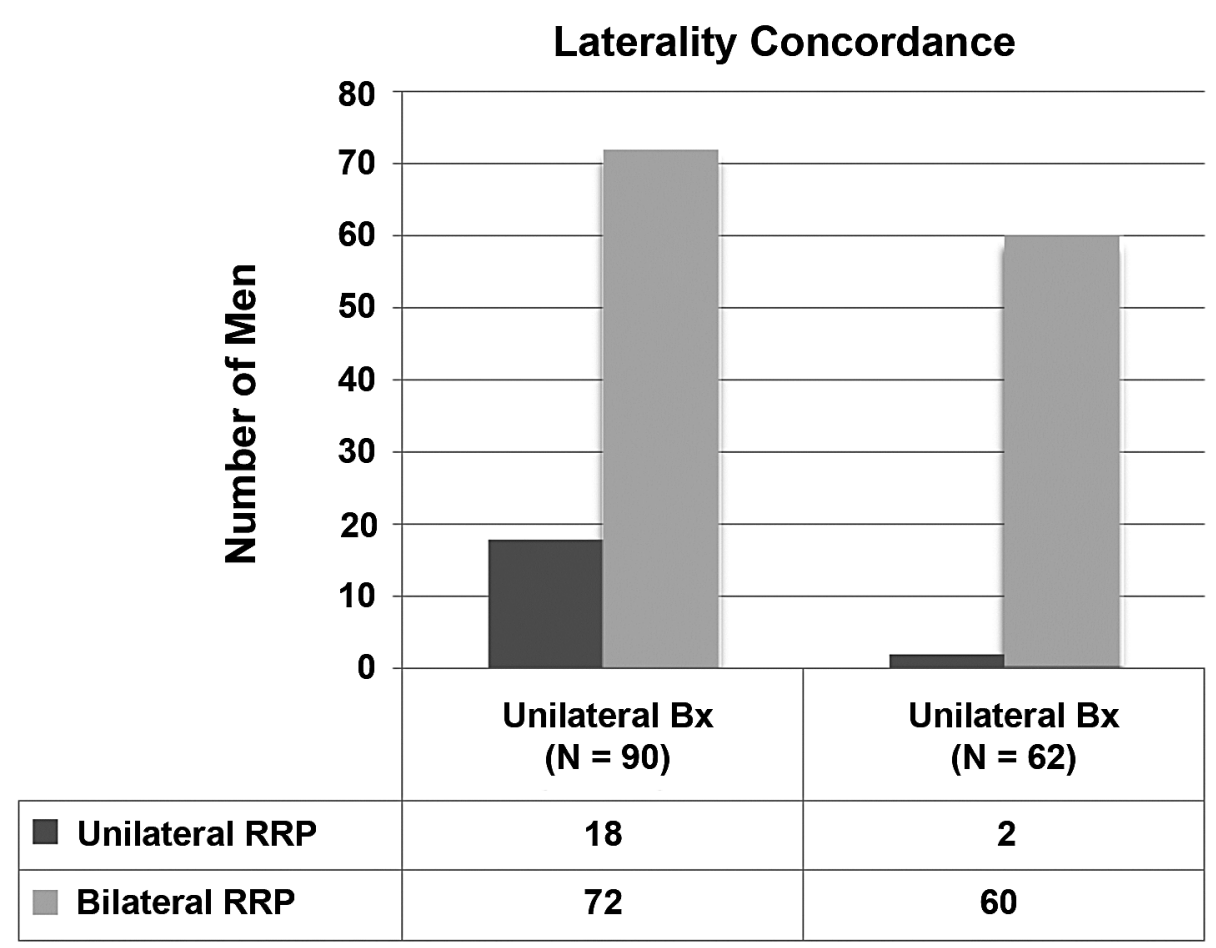

Figure 2 - Laterality concordance on radical retropubic prostatectomy (RRP).

on biopsy. These differences may be due to sampling error, pathologist's interpretation, or disease progression (8).

In our study, there was a general trend toward increased concordance when more prostate core biopsies were performed as the concordance rate was lowest in the 5-6 core group. Prior studies have suggested that taking more prostate cores may improve the concordance between biopsy and prostatectomy
(2-5), while others have argued that more cores does not improve prognostic information (6). Coogan et al. reported 10 cores provided an accuracy of $58 \%$ versus $40-41 \%$ in those with 8 and 6 cores (4). San Francisco et al. reported an extended biopsy regimen with 10 or more cores improved concordance from $63 \%$ to $76 \%$, and also proposed that processing each prostate core separately also improved accuracy (3). Similarly, Mian et al. noted improvement in accuracy

Table 3 - Effect of number of cores on concordance.

\begin{tabular}{lcc}
\hline Number of Core Biopsies & Number of Patients & Concordance \\
\hline $5-7$ & 32 & $34 \%$ \\
$8-9$ & 70 & $60 \%$ \\
10-12 & 46 & $46 \%$ \\
p Value for comparisons: & & \\
5-7 vs. $8-9(0.03)$ & & \\
$8-9$ vs. $10-12(0.19)$ & & \\
5-7 vs. $10-12(0.45)$ & & \\
\hline
\end{tabular}


Table 4 - Gleason concordance per year.

\begin{tabular}{ccc}
\hline Year & Number of Patients & Concordance \\
\hline 2000 & 21 & $29 \%$ \\
2001 & 22 & $64 \%$ \\
2002 & 28 & $64 \%$ \\
2003 & 31 & $42 \%$ \\
2004 & 30 & $53 \%$ \\
2005 & 20 & $45 \%$ \\
\hline
\end{tabular}

from $48 \%$ to $68 \%$ when more than 6 cores were taken (2). One study of 100 consecutive prostatectomy specimens evaluated by core biopsy of the surgical specimens on the bench top reported that prostate cancer detection improved from $75 \%$ with sextant biopsies to $88 \%$ with an extended 14 core biopsy (9).

Despite the suggestion that taking more cores directly leads to increased accuracy, the relationship is likely multi-factorial. Toward the later years of our study, more prostate cores were taken and accuracy declined, but was still improved compared to taking sextant biopsies. One possible explanation for this finding would be the change in pathologists' interpretation of the biopsy specimens. Sampling error can also be a contributing factor.

There are limitations to our study, including those inherent in a retrospective analysis. There was a general trend toward taking more core biopsies during the study period; however this was not done with a standardized protocol. The pathology review for this study was not performed by a single pathologist, and during review of prostatectomy specimens the pathologist was not blinded to the initial biopsy involvement, which could have influenced interpretation.

\section{CONCLUSIONS}

Prostate biopsy underestimated prostatectomy Gleason score in $34 \%$ of men and bilateral involvement in $80 \%$ of those with unilateral disease on biopsy. Taking at least eight cores improves the accuracy of the prostate biopsy.

\section{ACKNOWLEDGEMENTS}

This study is supported in part by funding from the Center for Research in the Implementation of Innovative Strategies in Practice (CRIISP), United States Department of Veterans Affairs.

The views expressed in this article are those of the authors and do not necessarily reflect the policy or position of the Department of Veterans Affairs.

\section{CONFLICT OF INTEREST}

None declared.

\section{REFERENCES}

1. Jemal A, Siegel R, Ward E, Murray T, Xu J, Thun MJ: Cancer statistics, 2007. CA Cancer J Clin. 2007; 57: 43-66.

2. Mian BM, Lehr DJ, Moore CK, Fisher HA, Kaufman RP Jr, Ross JS, et al.: Role of prostate biopsy schemes in accurate prediction of Gleason scores. Urology. 2006; 67: 379-83.

3. San Francisco IF, DeWolf WC, Rosen S, Upton M, Olumi AF: Extended prostate needle biopsy improves concordance of Gleason grading between prostate needle biopsy and radical prostatectomy. J Urol. 2003; 169: 136-40.

4. Coogan CL, Latchamsetty KC, Greenfield J, Corman JM, Lynch B, Porter CR: Increasing the number of biopsy cores improves the concordance of biopsy Gleason score to prostatectomy Gleason score. BJU Int. 2005; 96: 324-7.

5. Makhlouf AA, Krupski TL, Kunkle D, Theodorescu D: The effect of sampling more cores on the predictive accuracy of pathological grade and tumour distribution in the prostate biopsy. BJU Int. 2004; 93: 271-4.

6. Grossklaus DJ, Coffey CS, Shappell SB, Jack GS, Cookson MS: Prediction of tumour volume and pathological stage in radical prostatectomy specimens is not improved by taking more prostate needle-biopsy cores. BJU Int. 2001; 88: 722-6.

7. Carter HB, Kettermann A, Warlick C, Metter EJ, Landis P, Walsh PC, et al.: Expectant management 
of prostate cancer with curative intent: an update of the Johns Hopkins experience. J Urol. 2007; 178: 2359-64; discussion 2364-5.

8. Gleason DF: Histologic grading of prostate cancer: a perspective. Hum Pathol. 1992; 23: 273-9.
9. Nesrallah L, Nesrallah A, Antunes AA, Leite KR, Srougi M: The role of extended prostate biopsy on prostate cancer detection rate: a study performed on the bench. Int Braz J Urol. 2008; 34: 563-70; discussion 570-1.

Accepted after revision:

May 25, 2009

\section{Correspondence address:}

Dr. Fadi N. Joudi

Dept. of Urology, University of Iowa

200 Hawkins Dr., 3 RCP

Iowa City, IA 52242-1089, USA

Fax: + 1319 356-3900

E-mail: fadi-joudi@uiowa.edu 